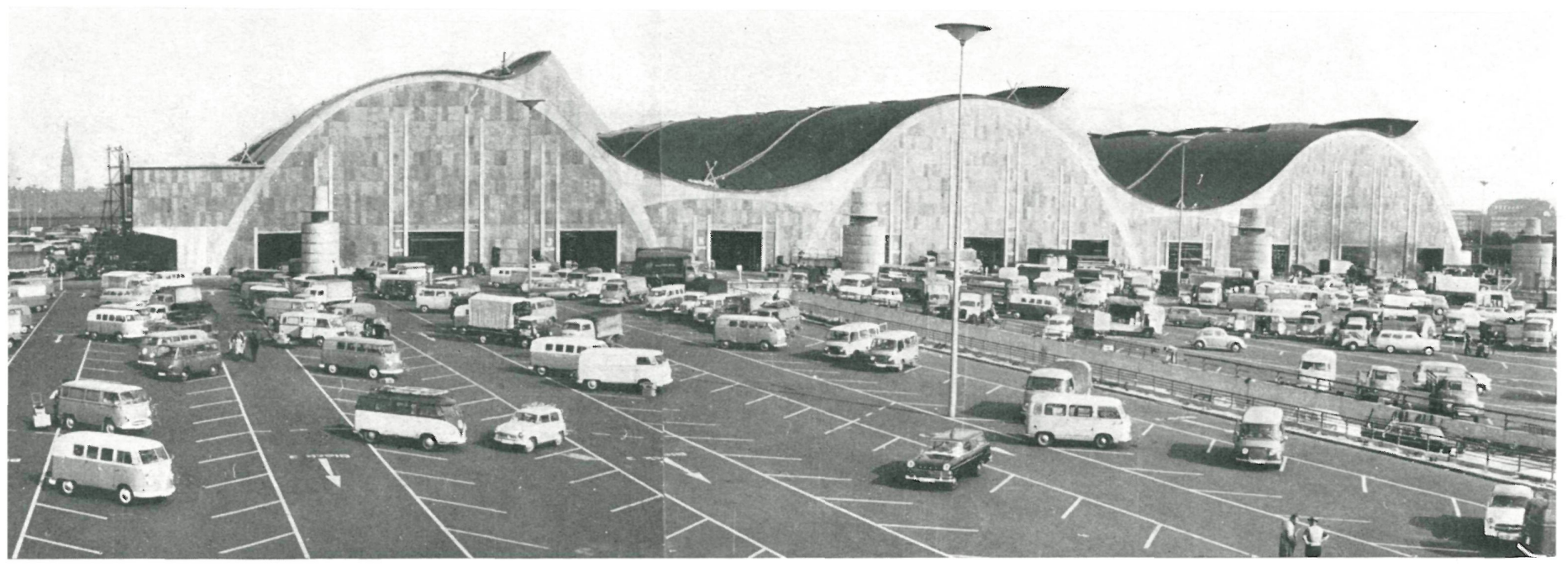

\title{
el nuevo mercado de Hamburgo
}

B. HERMKES, G. SCHRAMM y J. ELINGIUS, dipls. ingenieros

$136 \cdot 4$

\section{simopsis}

La nave-motivo de este artículo-, zonas de estacionamiento, muelles de descarga, playa de vías, sótanos para cámaras frigoríficas, asentamientos, servicios e instalaciones modernas hacen de este mercado un conjunto que es orgullo de la ciudad.

La cubierta, que constituye la parte de mayor interés, es una lámina, de hormigón armado y pretensado, de perfil ondulado con zonas suspendidas en los valles y lucernarios en las crestas de la misma.

La nave se subdivide en cinco crujías: tres de $48 \mathrm{~m}$ de luz y dos intermedias de $16 \mathrm{~m}$. Los arcos contiguos de las tres crujías principales se han arriostrado con piezas arqueadas, de pequeña flecha, que proporcionan gran rigidez al conjunto.

La construcción se realizó por trozos de $20 \mathrm{~m}$, partiendo del lado de $180 \mathrm{~m}$, en dirección normal, hasta llegar a los $220 \mathrm{~m}$, que tiene el otro lado del rectángulo que forma la planta. Se necesitaron dos meses para la construcción de un trozo de $20 \mathrm{~m}$, tiempo que se aprovechaba para ir preparando la infraestructura del trozo inmedia $:$

La ejecución se llevó a cabo empleando maquinaria auxiliar moderna, la cual permitió mantener el automatismo con el menor número posible de productores.

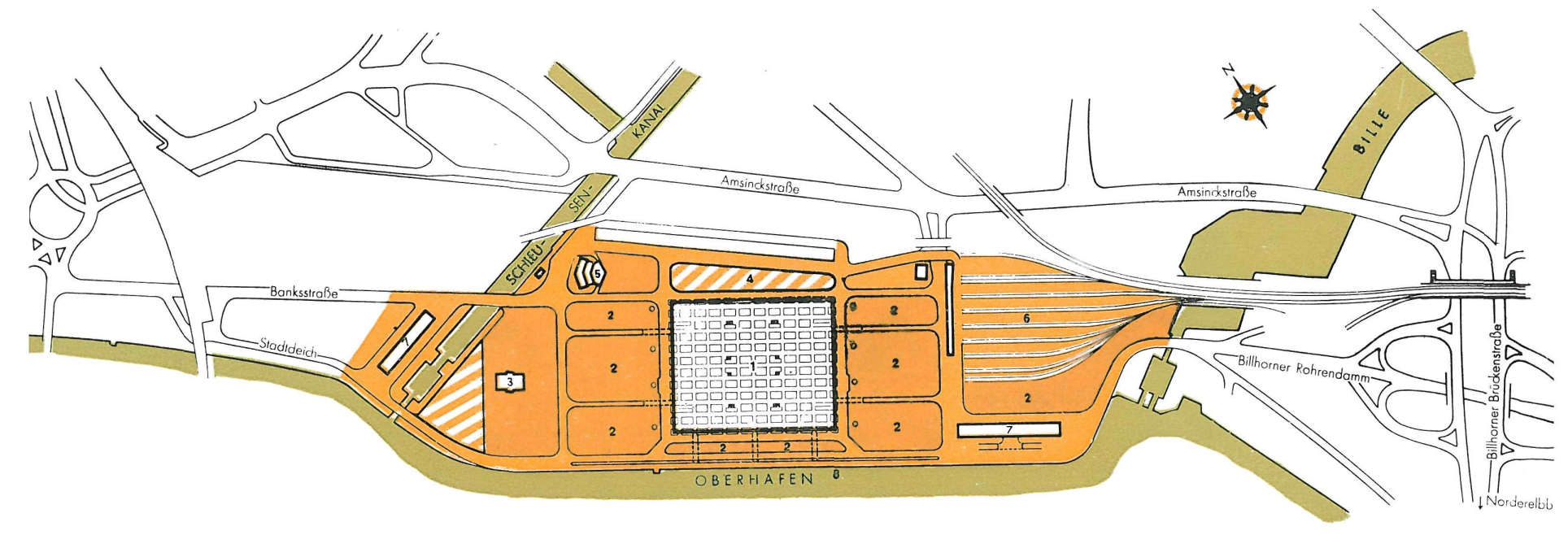

1. Nave central.-2. Estacionamiento.-3. Templete de subastas.-4. Asentadores.-5. Ofici nas.-6. Playa de vías.-7. Envases vacíos.-8. Muelles de descarga. 


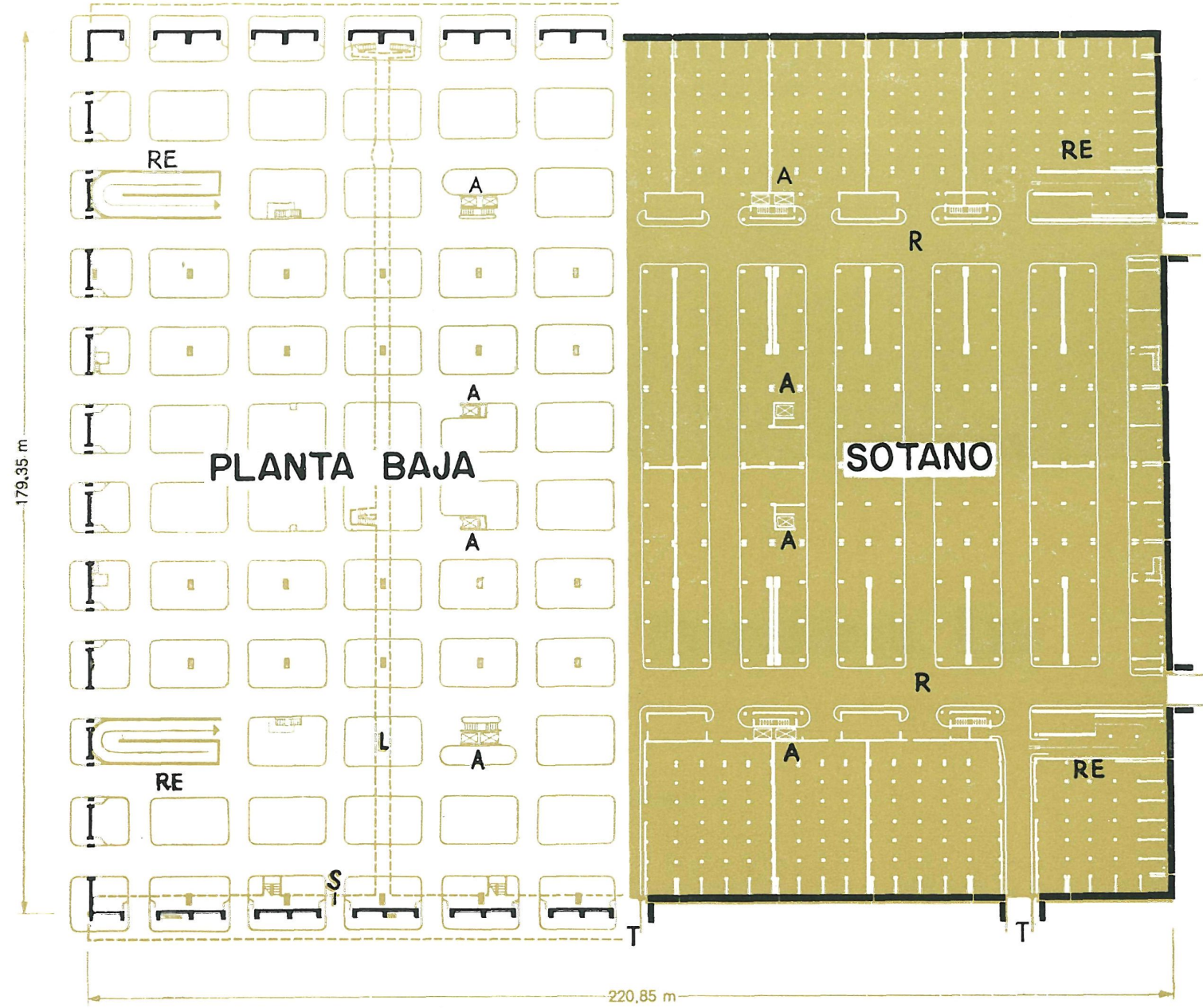

planta

A. Ascensores (montacargas).-L. Pasarela.-RE, Rampa para vehículos.

El mercado recientemente construido en Hamburgo (Alemania), cuya estructura fue proyectada por los autores de este trabajo, es el más importante de la ciudad.

La nave, de $220 \times 180 \mathrm{~m}$ en planta, va cubierta con una lámina de generatriz ondulada, de hormigón armado y pretensado y espesor variable. Los tres arcos principales forman otras tantas naves, mientras que los dos arcos, de concavidad invertida y que se unen a los anteriores, crean dos naves secundarias.

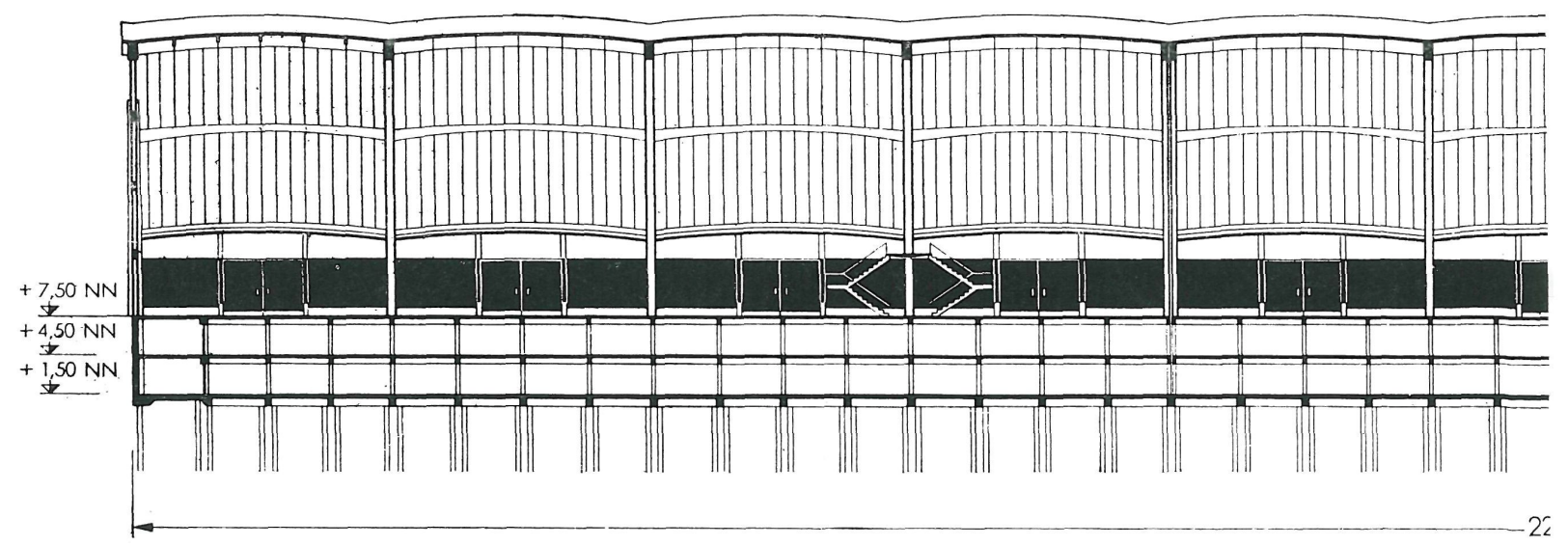



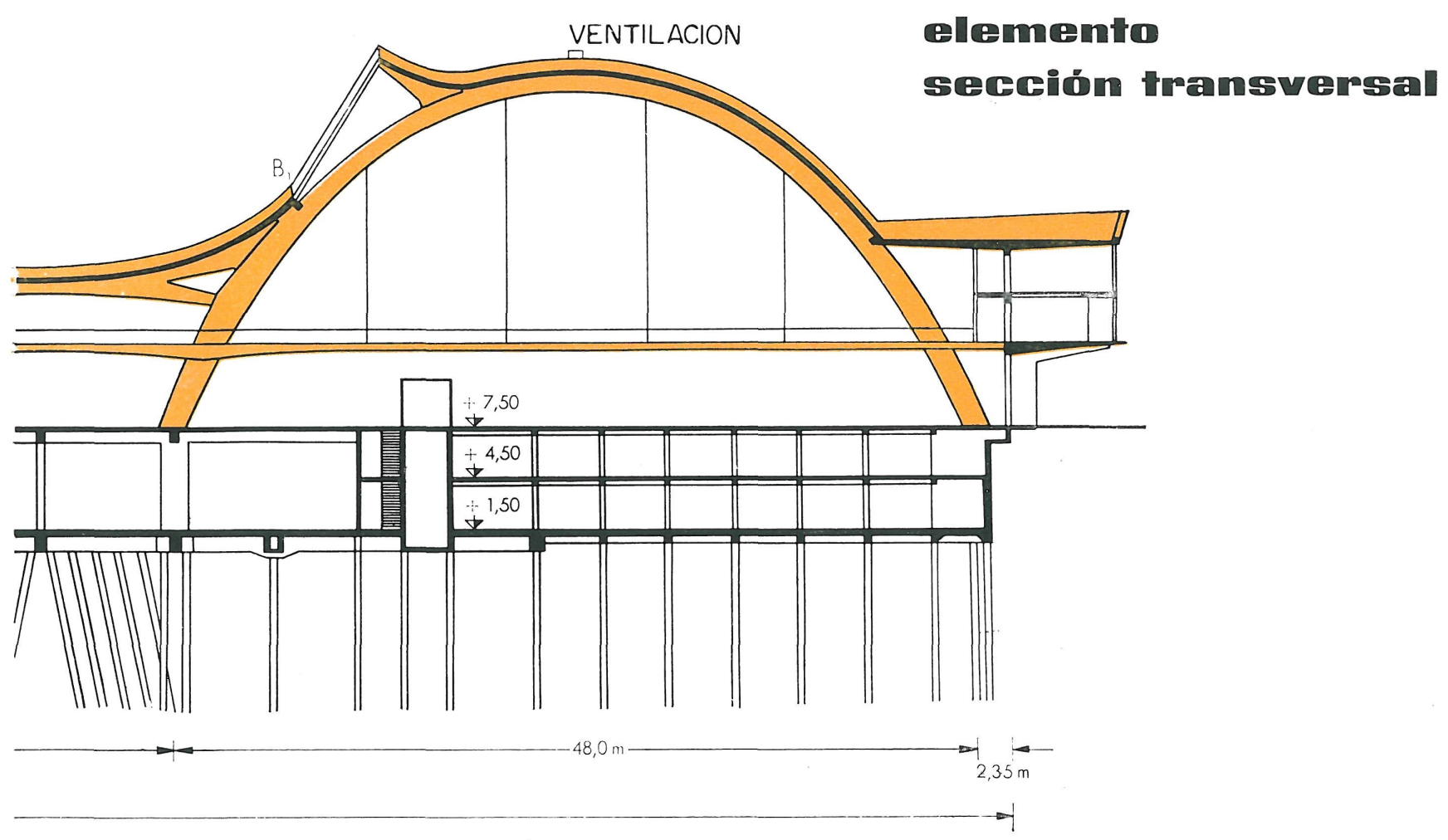

El edificio está dotado de toda clase de servicios e instalaciones modernas, que permiten conservar, madurar y transportar los productos alimenticios con facilidad y prontitud. Como los vapores, humos y atmósfera viciada son agentes evidentemente nocivos, la ventilación ha sido estudiada con gran interés para asegurar un ambiente sano $\mathrm{e}$ inodoro. Otro problema importante fue el de la iluminación natural, que se resolvió elegantemente mediante la introducción de lucernarios de $18 \times 180 \mathrm{~m}$, por lo que la forma exterior aparente de la cubierta es muy similar a lo que se ha dado en llamar "shed», actualmente, pero que siempre se le conoció por el nombre de diente de sierra.

El mercado, con sus servicios, superficie para el estacionamiento de 3.000 vehículos, recinto cerrado, proximidad al puerto, muelles de carga y descarga, sótanos, cámaras frigoríficas y nave destinada a la subasta de frutas y verduras, constituye un edificio modelo en su género.

\section{Cimientos}

El terreno sobre el que se yergue este mercado presentaba escasa capacidad de sustentación, por cuya razón se decidió la hinca de unos 5.300 pilotes, de hormigón, formando sobre ellos un enrejado cuya retícula varía de acuerdo con la carga que se ha de

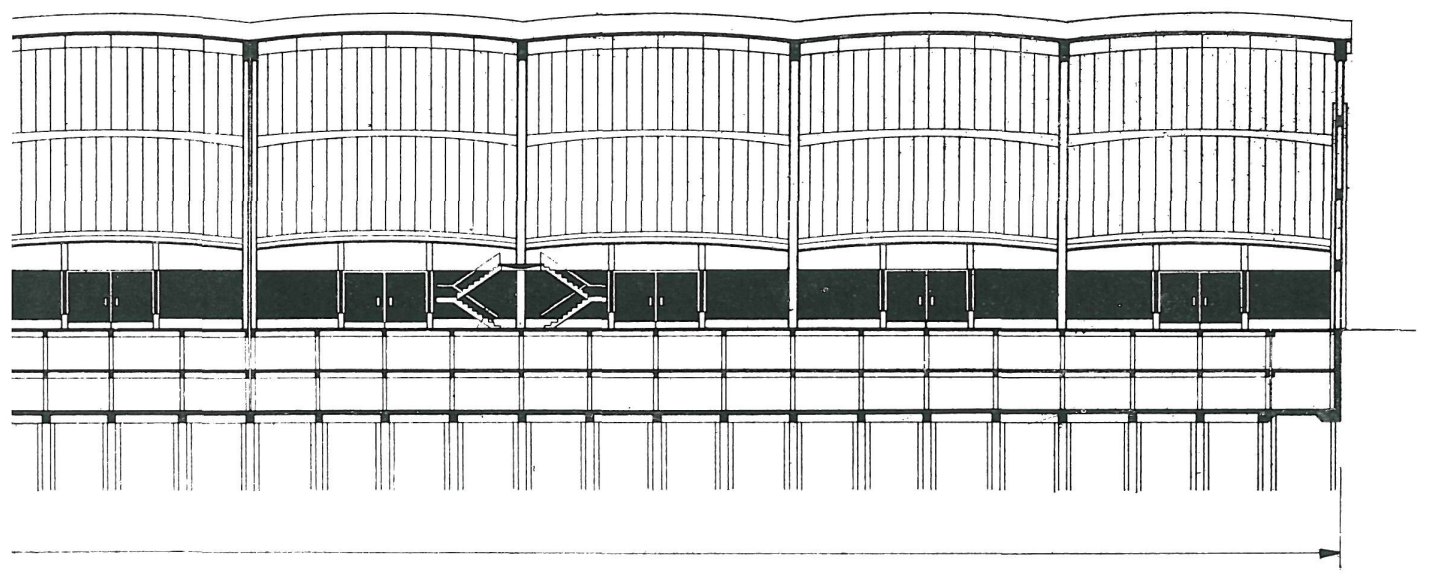

longitudimal 


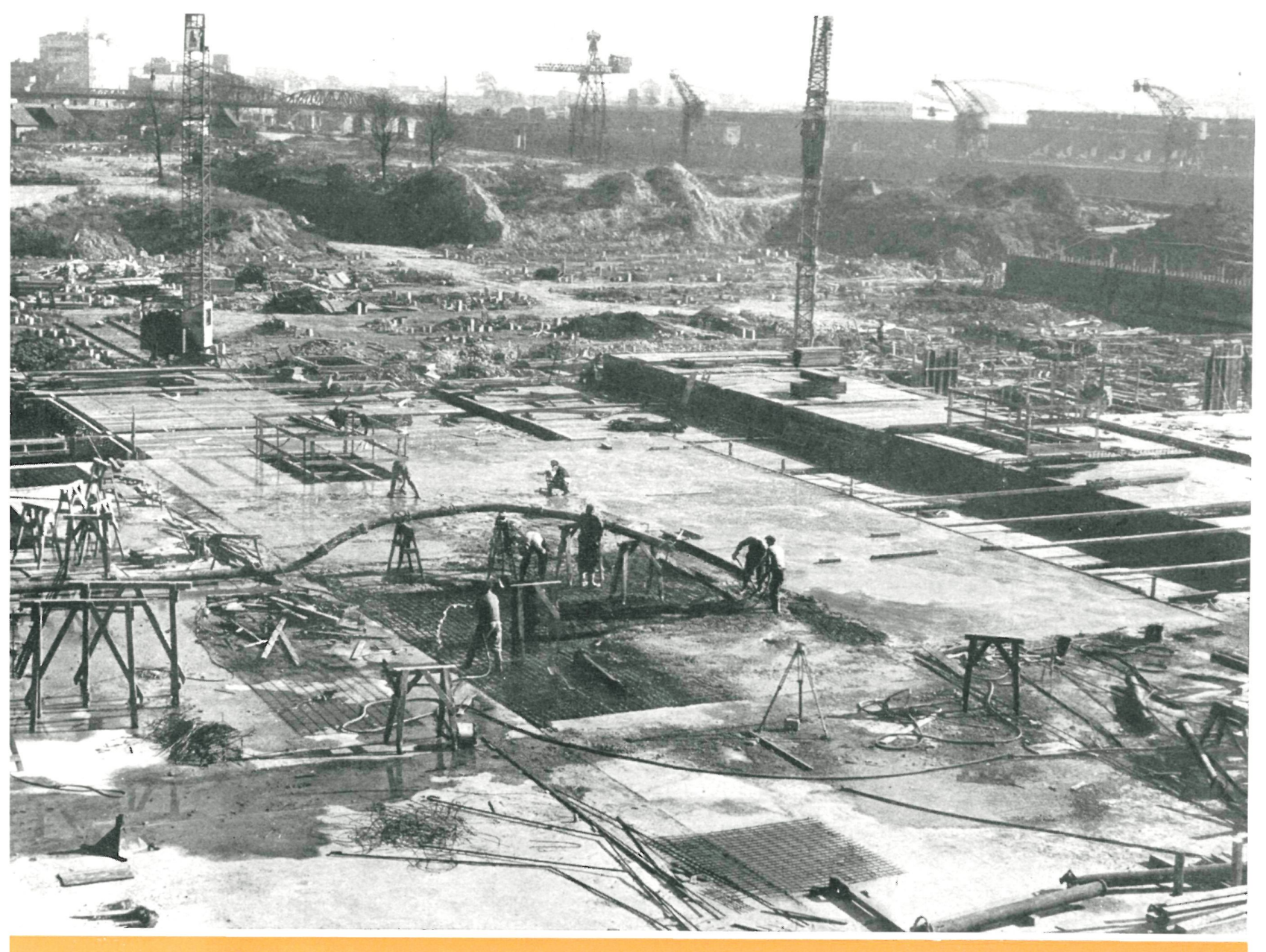

Hormigonado del forjado

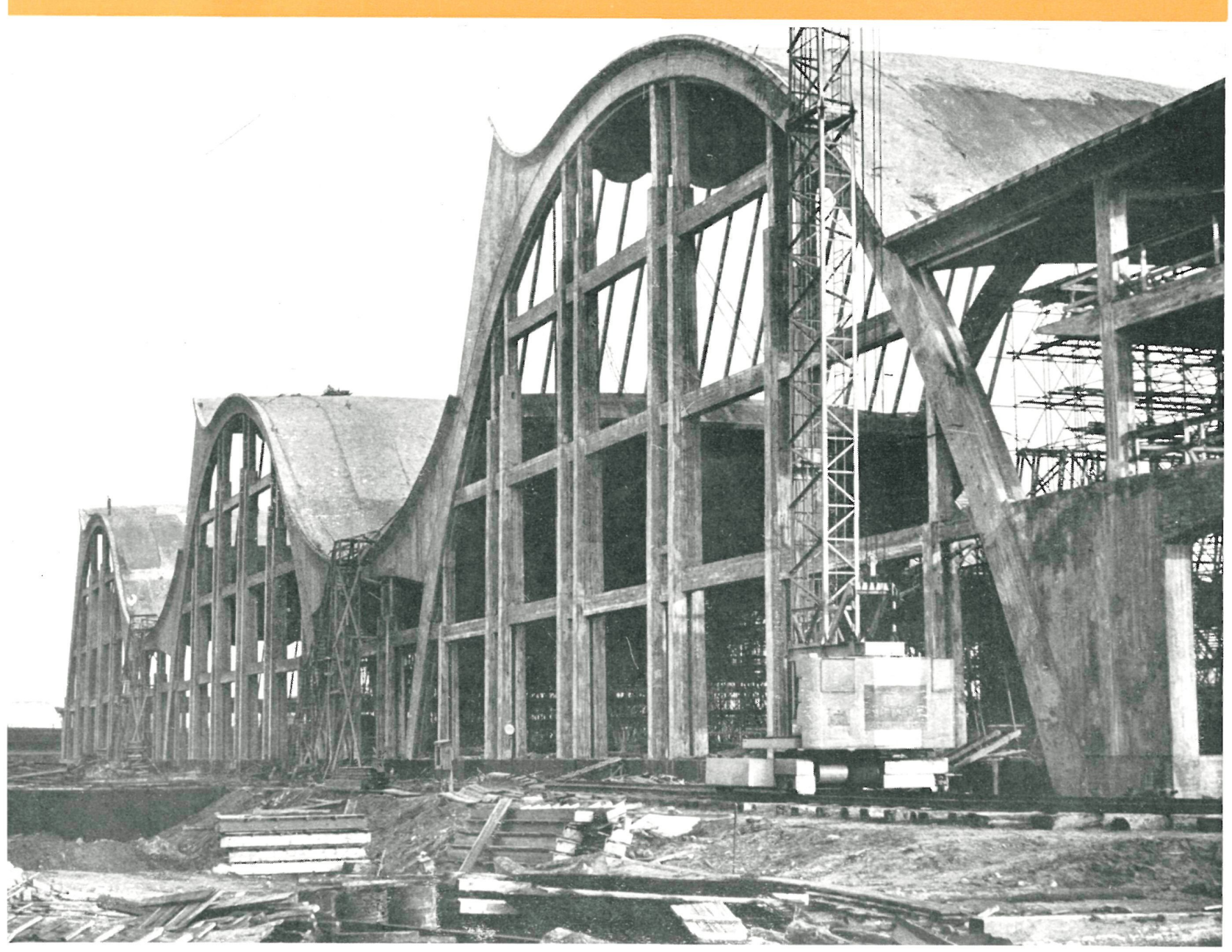


Vista general de la nave en construcción. Preparación de armaduras.

Entreplantas en una fachada con voladizo.

Construcción de lucernarios.

transmitir a cimientos. En la zona de sótanos, de doble planta, el reticulado es de $4 \times 5 \mathrm{~m}$, y en los de una sola planta, de $8 \times 6$ metros.

Como el nivel inferior del sótano se encuentra más bajo que el freático, la losa del piso se ha impermeabilizado convenientemente para evitar posibles infiltraciones. El forjado del sótano está constituido por una losa de hormigón armado, pretensada en sentido diagonal.

\section{Cubiemta Iamincen}

Como anteriormente se dijo, la cubierta consiste en una lámina, de hormigón $\mathrm{y}$ de forma ondulada que cubre cinco naves, con luces de $48 \mathrm{~m}$ las tres más importantes y de $16 \mathrm{~m}$ las dos restantes. La parte superior correspondiente a las naves mayores se ha pretensado siguiendo el sistema Dywidag. La lámina extrema orientada al norte termina en un lucernario, corrido de $18,30 \mathrm{~m}$ de altura; en las otras láminas, su altura es de 10 metros.

La cubierta está soportada por arcos de hormigón armado, de forma parabólica, espaciados a $20 \mathrm{~m}$. La lámina tiene un espesor que varía desde $13 \mathrm{~cm}$ en arranques a $8 \mathrm{~cm}$ en la clave, quedando suspendida en el tramo que sirve de enlace a las dos naves próximas de mayor luz.

Entre los arcos correspondientes a dos naves sucesivas se han construido unas piezas transversales, arqueadas, de hormigón armado, que aseguran la estabilidad y arriostramiento entre los arcos de apoyo de la lámina de cubierta. Estas piezas en arco tienen unos $60 \mathrm{~cm}$ de flecha y $50 \mathrm{~cm}$ de canto.

La flecha de las láminas de mayor altura es de $21 \mathrm{~m}$ respecto al suelo del mercado, mientras que las invertidas o suspendidas tienen una altura de $8,5 \mathrm{~m}$ entre su parte inferior y el referido suelo.
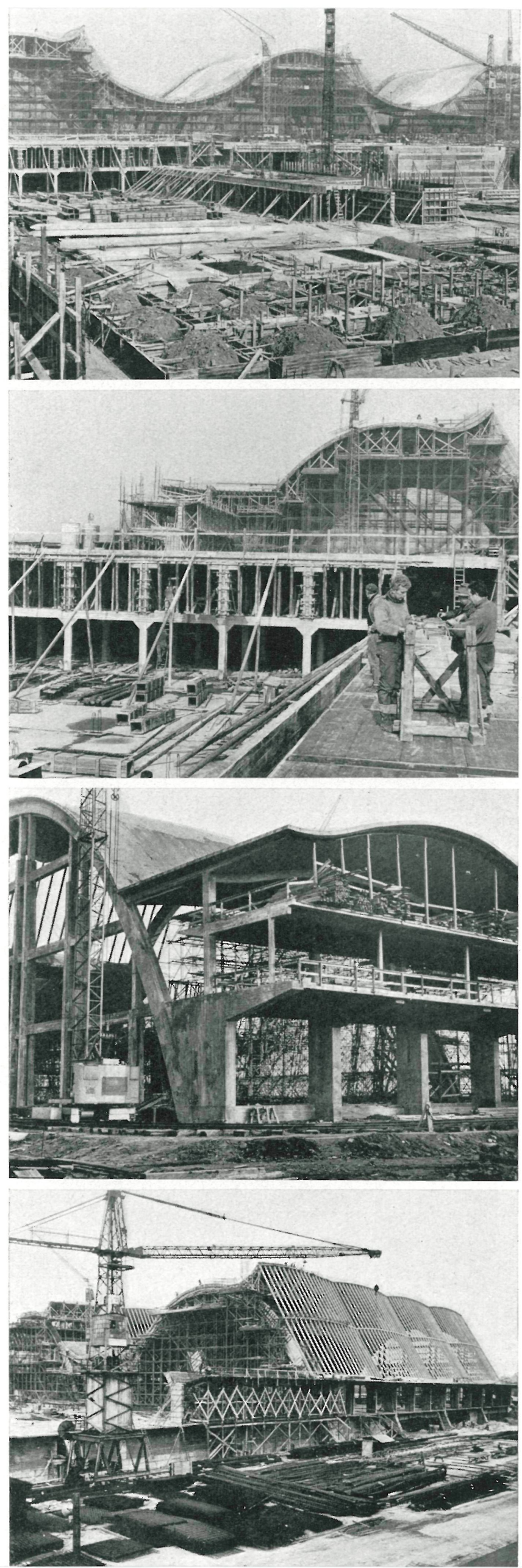


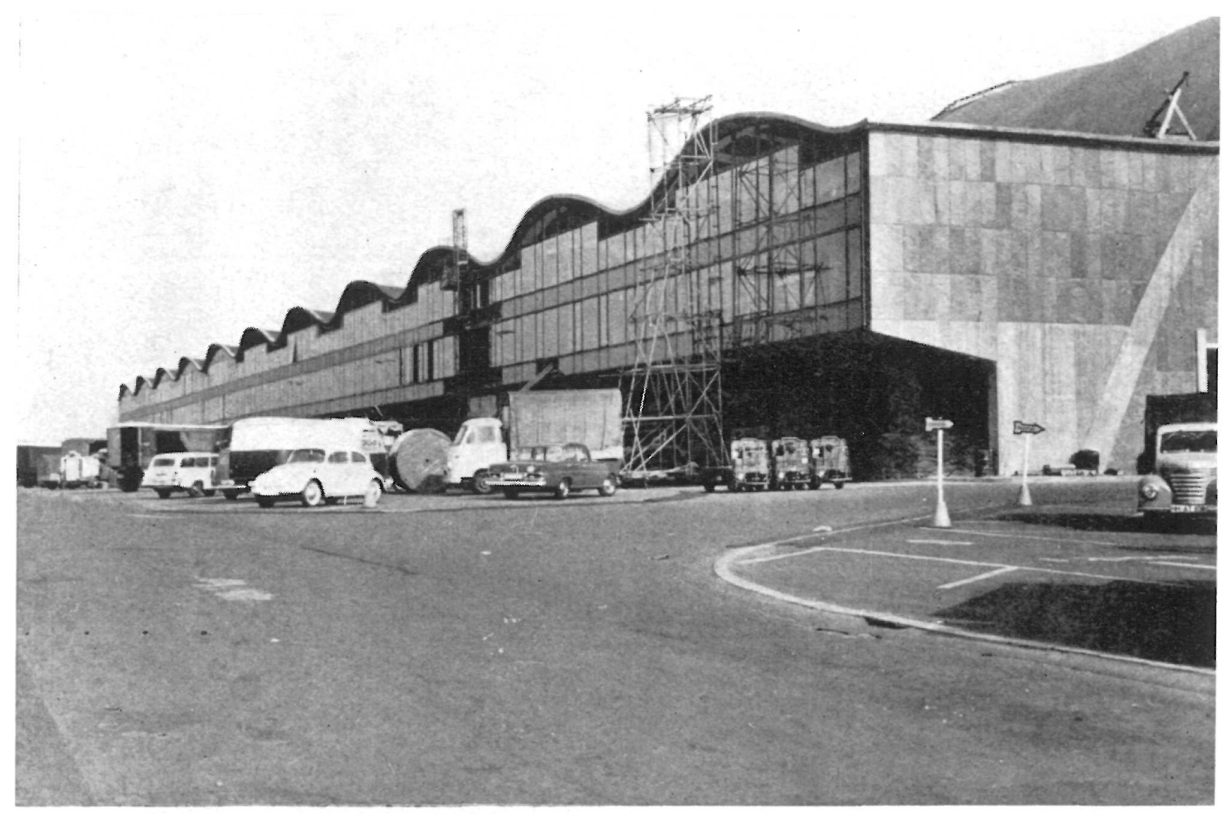

Muelles bajo el voladizo.

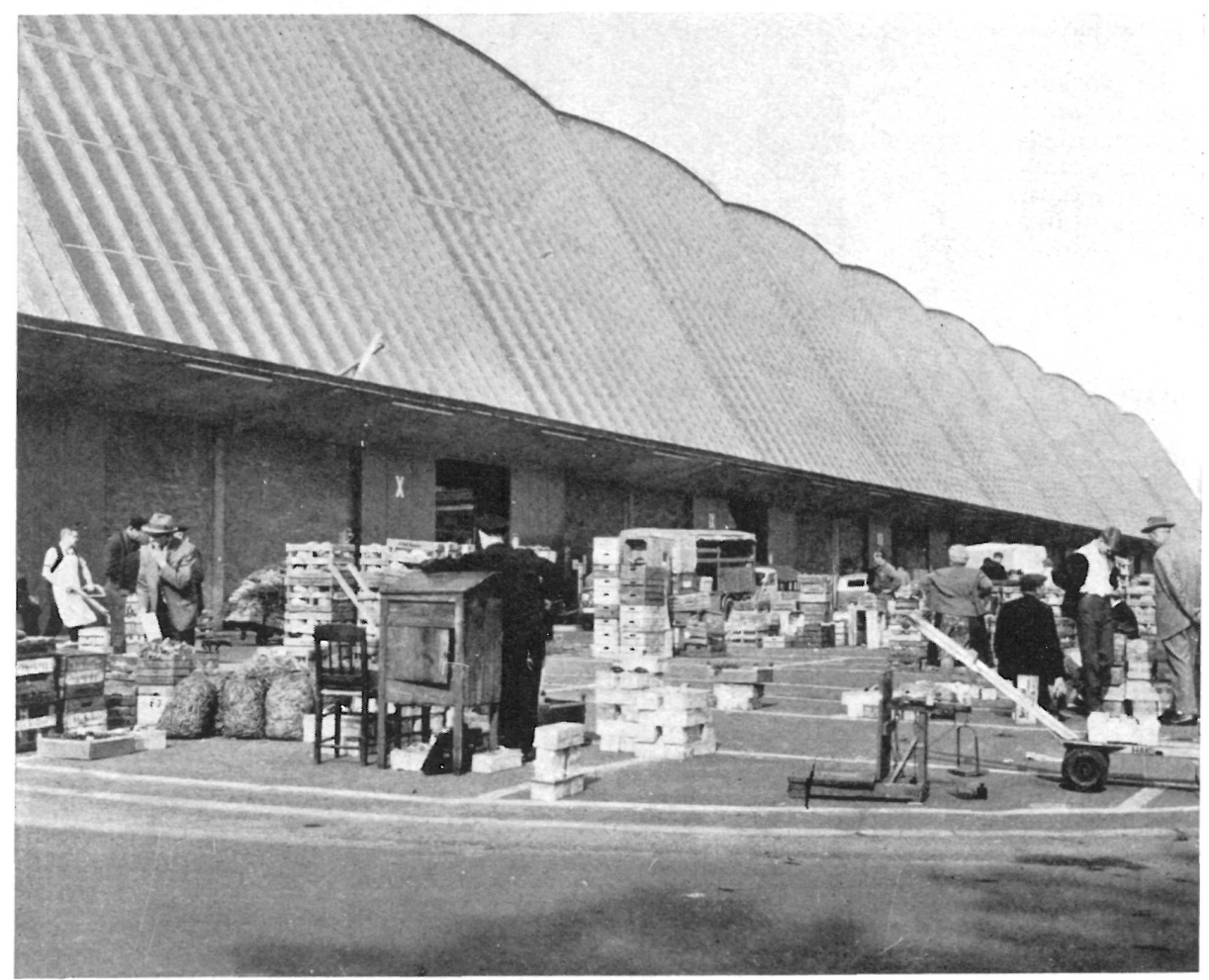

Asentamiento de mercancías.

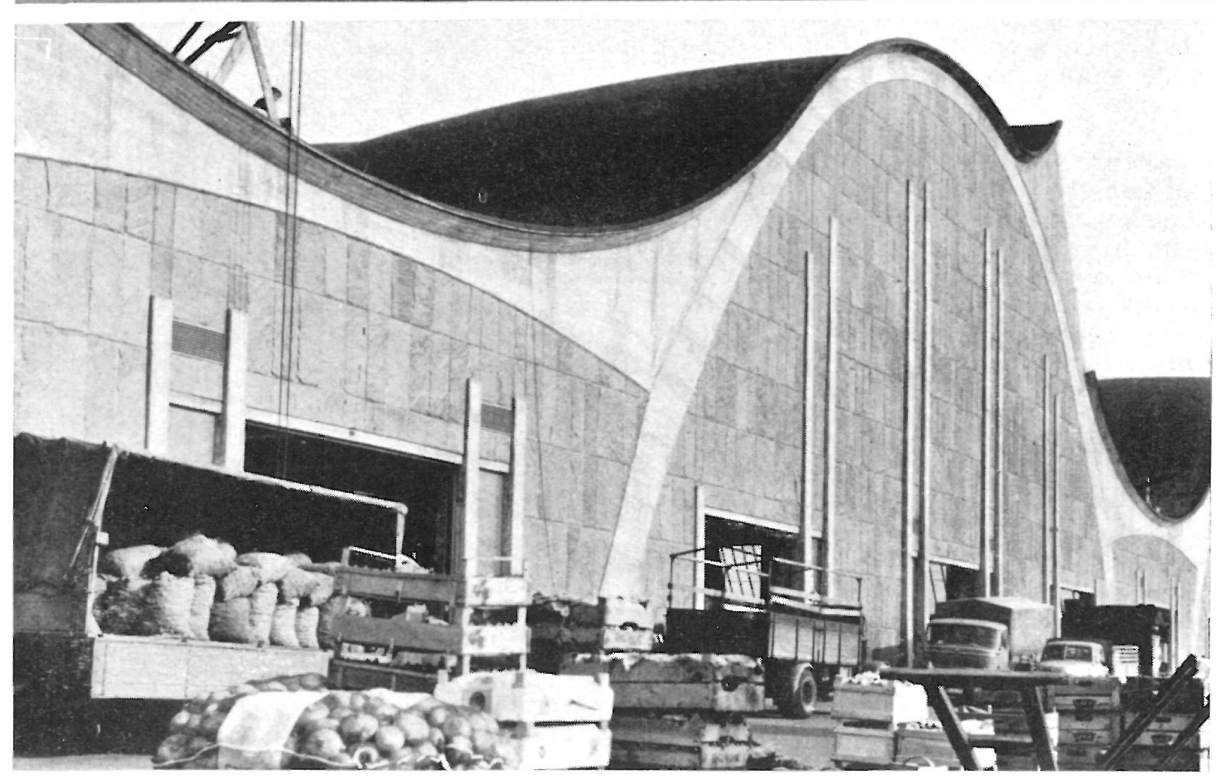

Movimiento de mercancías. 
Con objeto de aislar e impermeabilizar lo mejor posible la cubierta, se revistió, en su trasdós, con dos capas de asfalto especial aplicado sobre una malla de alambre.

\section{Ejecureión}

La nave se construyó por trozos sucesivos de $20 \mathrm{~m}$ y a partir del lado corto de $180 \mathrm{~m}$, con dirección normal, hasta terminar los $220 \mathrm{~m}$, que mide el lado largo del rectángulo de la planta.

Este sistema de construcción por trozos sucesivos permitió ir preparando cimientos y sótanos de cada uno mientras se terminaba el anterior. La construcción de cada tramo de $20 \mathrm{~m}$ requirió un período de tiempo de 2 meses.

La construcción del mercado corrió a cargo de las empresas Dyckerhoff \& Widmann KG y Hermann Möller Siemens. Bauunion GmBh Lenz-Bau Ag., que pusieron a contribución todo el automatismo y medios auxiliares necesarios, gracias a lo cual el numero de productores en el momento de mayor actividad no pasó de unos 380 .

Adaptado por J. J. Ugarte.

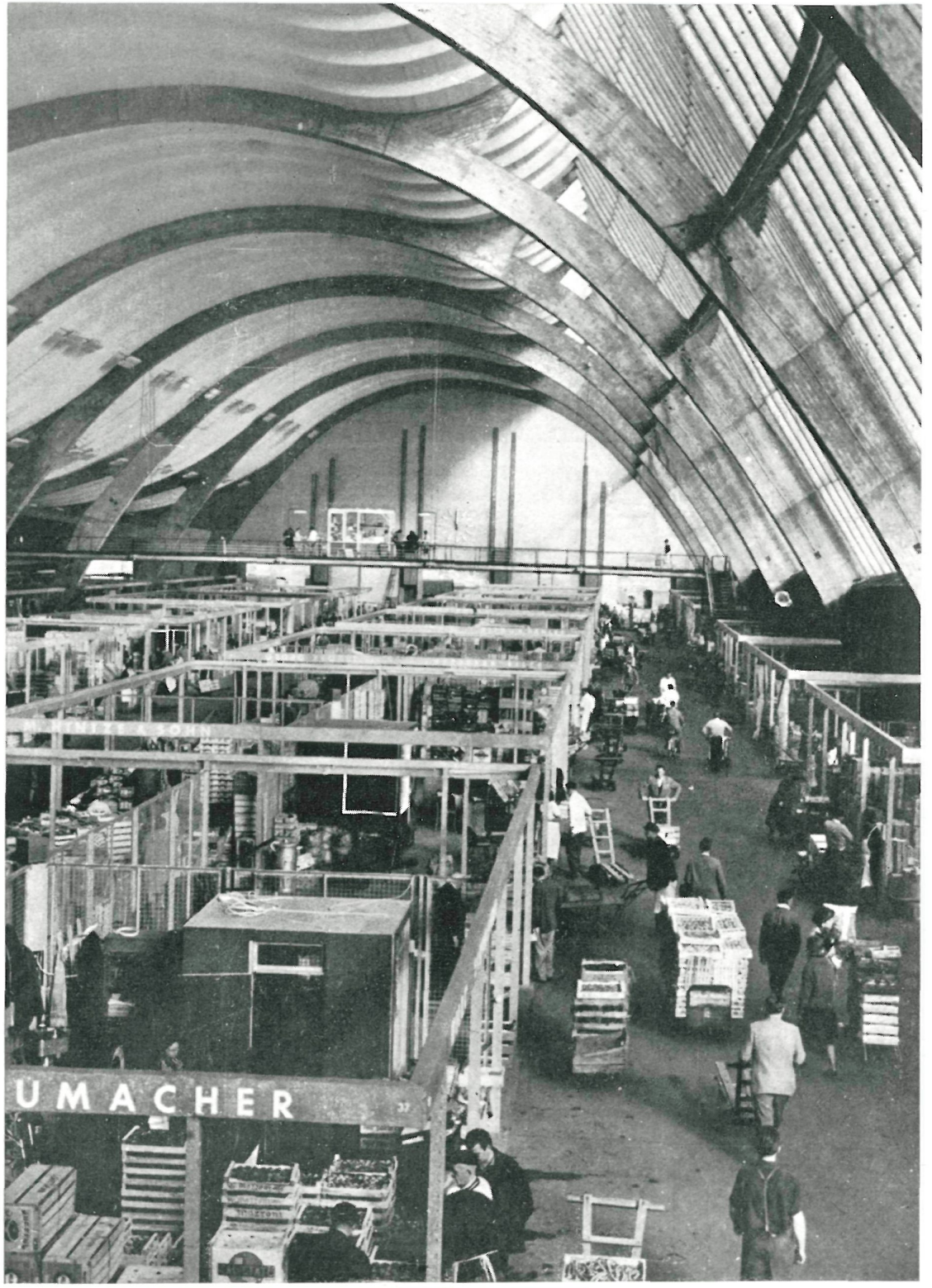

Vista interior de

una nave.

Escalera de hormigón armado.

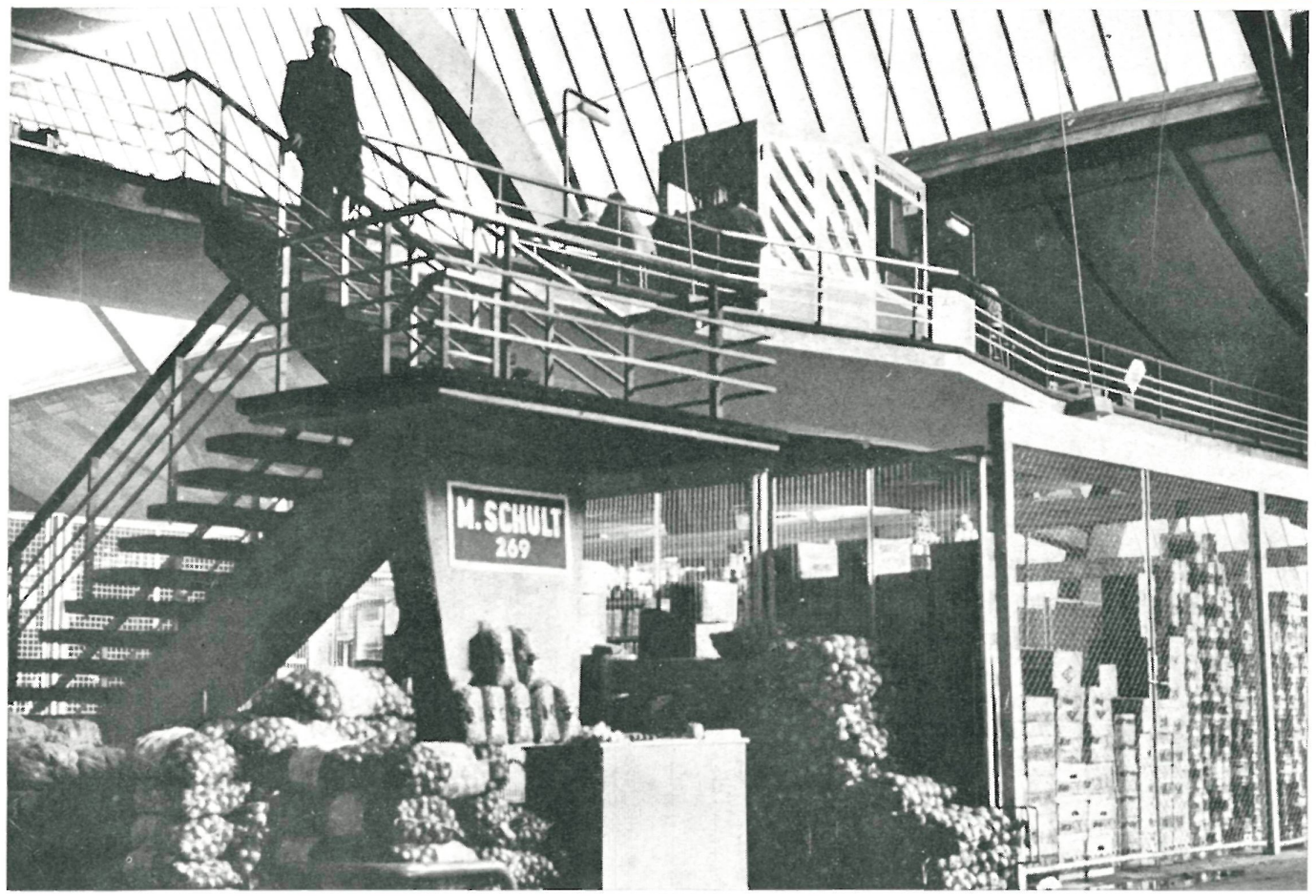

ST: LICHTBILDNEREI DER BAUBEHORDE, HAMBURG 


\section{Le mouvedu unathé d" Hambourg}

B. Hermkes, G. Schramm et J. Elingius, ingénieurs diplômés

Le hall-objet de cet article-, les zones de stationnement, quais de déchargement, installations ferroviaires, sous-sols pour les chambres frigorifiques, services et installations modernes font de ce marché un ensemble orgueil de la ville.

Le couverture, qui constitue la partie la plus intéressante, est un voile mince, en béton armé et précontraint, de profil ondulé avec des zones suspendues dans les vallées et des lucarnes à leurs sommets.

Le hall se divise en cinq travées, trois de $48 \mathrm{~m}$ de portée et deux autres intermédiaires, de $16 \mathrm{~m}$. Les arcs contigus aux trois travées principales ont été contreventés à l'aide de pièces arquées, de peu de flèche, qui assurent la bonne rigidité de l'ensemble.

La construction a été réalisée par tronçons de $20 \mathrm{~m}$, partant du côté de $180 \mathrm{~m}$, en direction normale, jusqu’à parvenir aux $200 \mathrm{~m}$ de longueur que compte l'autre côté du rectangle formé par le plan. Deux mois ont été nécessaires pour la construction de chaque troncon de $20 \mathrm{~m}$, laps de temps utilisé pour préparer l'infrastructure du tronçon immédiat.

L'exécution a été réalisée grâce à l'emploi d'engins auxiliaires modernes, qui ont permis de maintenir l'automatisme avec le moins de main-d'oeuvre possible.

\section{The mew Hamburgh monolket}

B. Hermkes, G. Schramm and J. Elingius, diploma engineers

This article deals specially with the parking hall, the unloading platforms, the railway network, the undergound cooling chambers and the ancillary services which make this a most modern market; and a source of pride to Hamburg.

The roof structure is of special interest, and consists of a reinforced and prestressed concrete shell, of on. dulated outline. The synclines are suspended, and the anticlynes of the shell contain skylights.

The nave is subdivided into five longitudinal sections, three of them $48 \mathrm{~m}$ in span and two intermediate ones, of $16 \mathrm{~m}$ width. The arches covering the three main sections are interconnected with slightly arched elements, which provide great stiffness to the whole roof structure.

The construction of this roof was done in stages of $20 \mathrm{~m}$ length each, and $180 \mathrm{~m}$ width, and advanced progressively along the $220 \mathrm{~m}$ length of the nave. Each stage took 2 months to complete, and in this time the infrastructure of the next stage was prepared.

Work was done with the aid of modern construction machinery, and this made it possible to mechanise most of the operations, and employ a small number of operators.

\section{Die meut Markthalle im Hamburg}

B. Hermkes, G. Schramm und J. Elingius, Dipl. Ingenieure

Die Halle, Anlass zu diesem Aufsatz, ferner Parkmöglichkeiten, Entladeschuppen, Abstellgleise, Unterkellungen für Kühlkammern, Plätze für die Grosshändler, moderne Waschräume und sonstige Einrichtungen lassen diese Markthalle zu einem sehenswerten Bauwerk der Stadt werden.

Das Dach, der interessanteste Teil des Bauwerkes, besteht aus einer wellenförmigen Stahl- und Spannbetonplatte und ist hängend in den unteren Wölbungen, während es Oberlicht an den höchsten Stellen derselben hat.

Die Halle ist in fünf Gänge unterteilt: 3 davon mit $48 \mathrm{~m}$ lichter Weite und 2 dazwischenliegende mit je $16 \mathrm{~m}$ lichter Weise. Die Bogen der drei Hauptgänge sind durch flachgewölbte Verstrebungen verbunden, die dem Ganzen eine grosse Festigkeit verleihen.

Der Bau erfolgte in Abschnitten von je $20 \mathrm{~m}$, rechtwinklig, angefangen bei der $180 \mathrm{~m}$ langen Seite und beendet bei $220 \mathrm{~m}$ der langen Seite des im Rechteck aufgeführten Baues. Jeder Abschnitt von $20 \mathrm{~m}$ brauchte zwei Monate, in welcher Zeit der Unterbau des nächstfolgenden Abschnittes hergestellt wurde.

Unter Einsatz von modernen Hilfsmaschinen erfolgten die Arbeiten weitestgehend automatisch, so dass die menschliche Arbeitskraft auf ein Minimum beschränkt werden konnte. 